\title{
Sequential soil washing techniques using hydrochloric acid and sodium hydroxide for remediating arsenic-contaminated soils in abandoned iron-ore mines
}

\author{
Min Jang , Jung Sung Hwang , Sang II Choi
}

Introduction

About 894 of 900 abandoned metal mines are creating significant environmental problems in Korea. Mining waste and acid mine drainage produced from these abandoned metal mines have released several toxic metalloids or heavy-metals into ground-water, surface-water, and geological environments because of their solubility and mobility (Mulligan et al., 2001). There are two categories of processes that mostly control arsenic mobilities: (1) adsorption and desorption, and (2) solid-phase precipitation and dissolution. These processes can be mainly controlled by $\mathrm{pH}$, redox reactions, competing anions, and microbial activities (Kim et al., 2002). Among these factors, the pH

and redox reactions may be the most important parameters

to control the arsenic mobility through both processes, even though potential rates of the two processes are different. Arsenic and iron oxides have a redox-sensitive nature.

Therefore, transfer of large amounts of arsenic between the solid phases and neighboring water may result from redoxfacilitated precipitation and dissolution reactions (Camm et al., 2004). Arsenic dissolution can occur due to changes in the geochemical environment into a reductive condition. A high $\mathrm{pH}$ condition can also induce desorption of arsenic due to the negative net surface charge of iron oxide (Pfeifer et al., 2004). Since arsenic can be transported to other areas through these processes and can create secondary arseniccontamination sites, arsenic-contaminated soils must be treated in a rapid and safe way.

USEPA (1997) and Mulligan et al. (2001) described several 
available remediation technologies for heavy-metal contaminated soils. Among remediation technologies, soil washing cannot only extract heavy-metals or metalloids adsorbed or precipitated into soils, but it can also reduce the volume of contaminated soils. Soil washing can also be applied to large contaminated areas because of its rapid kinetics, ease, and economic efficiency (USEPA, 2001). For washing techniques, the selection of extractants (or agents) is the most important step because the extraction effectiveness of each extractant is different depending on its target contaminants, bonding strength, and soil characteristics. Up until now, several types of extractants (e.g., inorganic salts, inorganic acids, organic acids, and alkaline agents) have been studied for extracting heavy-metals or metalloids from tailings or soils. Among them, sodium hydroxide and hydrochloric acid have been known to be economical and effective for extracting arsenic from soils (Van Benschoten et al., 1994; Jang et al., 2005).

In this study, sequential washing techniques using single or dual agents ( $\mathrm{NaOH}$ and hydrochloric acid) were applied for arsenic-contaminated soils in an abandoned iron-ore mine area. Arsenic-containing iron-ore fines in this area can produce mobile colloids through coagulation and flocculation in natural waters contacting the soils (Pandey et al., 2004). The mobile colloids can be easily transported to other areas and can create secondary arsenic-contamination sites. Thus, a rapid and effective remediation is needed to satisfy the arsenic regulation for reuse or safe disposal of washed soils. Through establishing the following objectives, an effective washing strategy was found for treating arsenic-contaminated soils in floc-forming iron ore, as well as arsenic in the washing effluents. The specific objectives are as follows: (1) to observe the physico-chemical properties and arsenic partitioning into different compartments of soils through the sequential extraction procedure, (2) to determine parameters of soil washing such as effective physical sizing, types and concentration of washing agent, 
and ratio of agent volume to soil mass, (3) to find washing efficiencies with different types of sequential washing steps using single or dual agents and floc removal, and finally (4) to investigate remediation strategies to enlarge arsenic removal efficiencies for both soils in abandoned iron-ore mines and arsenic-containing washing effluents, while meeting the regulatory limit for washed soils.

Materials and methods

\subsection{Soil selection and characteristics}

Soil samples (designated as DC soils) were collected from arsenic-contaminated areas located at the Dal-Chun abandoned mine (Ulsan, Kyungsannamdo, South Korea) that had been developed for iron ore. Iron sources of the iron-ore mine mainly consist of the iron oxide minerals: magnetite $\left(\mathrm{Fe}_{3} \mathrm{O}_{4}\right)$ and hematite $\left(\mathrm{Fe}_{2} \mathrm{O}_{3}\right)$, goethite ( $\mathrm{Fe}_{2} \mathrm{O}_{3} Æ \mathrm{H}_{2} \mathrm{O}$ ), and limonite (a mixture of hydrated iron oxides). Magnetite is a naturally occurring metallic mineral that is the dominant species of magnetic compartments, while hematite and goethite are the main species of crystalline iron oxides, which are nonmagnetic minerals. The DC soils include both nonmagnetic and magnetic minerals (Fig. 1).

The arsenic concentration of this area was much higher than $15 \mathrm{mg} \mathrm{kg}_{-}$( $\mathrm{mg}$ of arsenic per $\mathrm{kg}_{-} 1$ of soils), which is the concern level of the Soil Environment Conservation Act of Korea (MOE, 2003) legislated by Korean Ministry of Environment (MOE). The Korean standard test (KST) methods were utilized to estimate arsenic concentrations extracted from soils. The total volume of arsenic-contaminated soils for this area was about 1780000 m3. Soils taken from this area were sieved through a $4.75-\mathrm{mm}$ opening sieve (no. 4) to remove large particles and allow a homogeneous soil size distribution. Characteristics of DC soils 


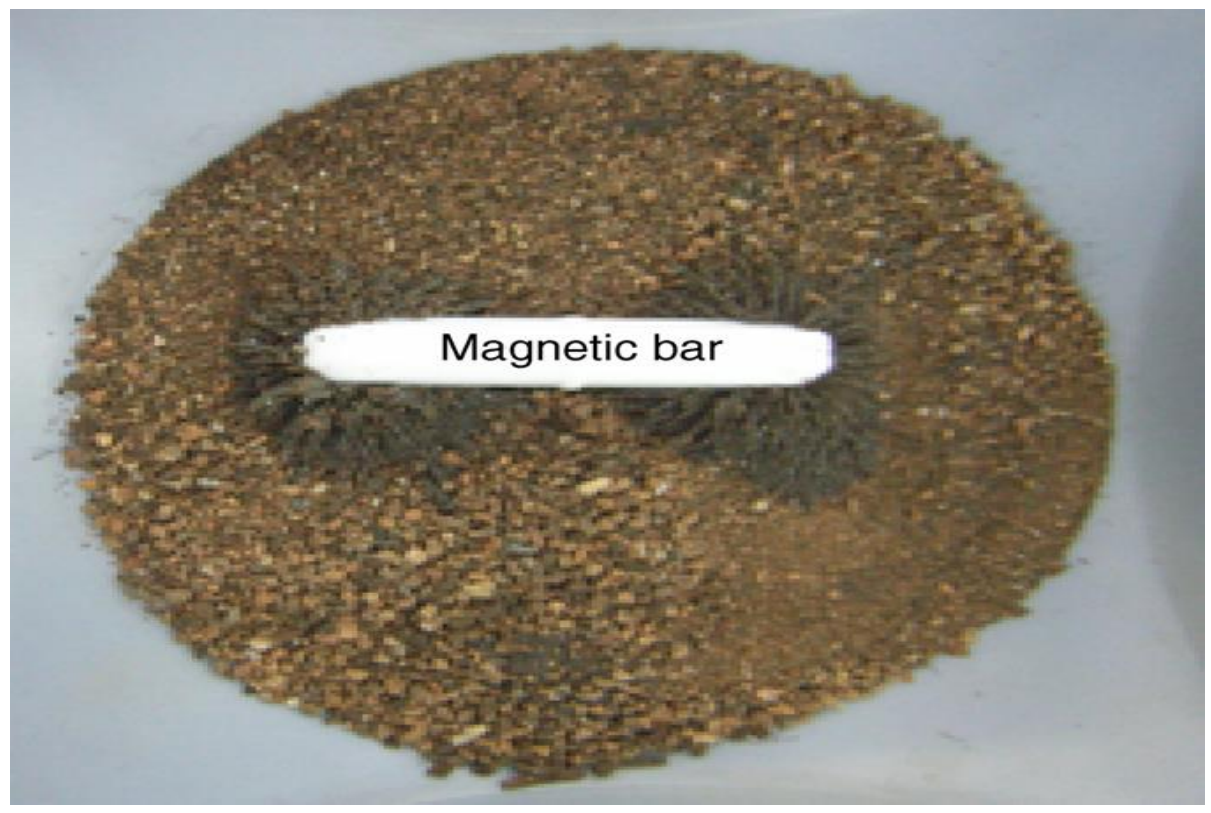

Fig. 1. Photo of DC soils: magnetic separation of magnetite.

such as $\mathrm{pH}$, organic content, particle density, and uniformity coefficient ( $\left.\mathrm{D}_{60} / \mathrm{D}_{10}\right)$ were measured by Methods of Soil Analysis (Page et al., 1986). The measurement of the cation-exchange capacity (CEC) was conducted by EPA Method 9080 (USEPA, 1986a,b). The total arsenic and iron concentrations of soil samples were measured by EPA 3050B (USEPA, 1986a,b), which is a hot nitric acid digestion method for soil. The detailed method of EPA 3050B adopted in this study was well described by Jang et al. (2005). As a disposal or reuse criterion of arsenic-contaminated soils, the KST methods for soils were adopted from the Soil Environment Preservation Act (MOE, 2002). The strictest regulation of arsenic concentration (6 mg kg_1) extracted by the KST methods was selected as a strategy of soil remediation. The KST method is as follows:

(1) add $50 \mathrm{ml}$ of $\mathrm{HCl}(1 \mathrm{M})$ to each $10 \mathrm{~g}$ of soil sample,

(2) shake the suspension at a speed of $100 \mathrm{rpm}$ and 30 _C for $30 \mathrm{~min}$, (3) centrifuge $10 \mathrm{ml}$ of suspension at $3200 \mathrm{rpm}$ for $20 \mathrm{~min}$, and (4) filter the supernatant with a 0.6-1m micropore filter, dilute the filtrate, and acidify the filtrate with conc. $\mathrm{HNO}_{3}$ before the arsenic analysis. Arsenic concentrations of filtrates were measured using inductively coupled plasma spectrometry (ICP-1000VI, 
Shimadzu Company, Japan) at a concentration range of 0.02-20 mg I_1. For the arsenic precipitation tests of washing effluents, arsenic concentration was measured by atomic absorption spectrometry (AA-6401F, Shimadzu_, Japan) connected with a continuous hydride generator (HVG-1, Shimadzu_, Japan) that has a detection limit of $0.5 \mathrm{lg} \mathrm{I}$. 1 . Duplicates, blank and EPA reference standards were analyzed with each set of samples as a quality control check on the analysis.

Arsenic and iron sequential extraction procedure, soil sieve analysis and arsenic extraction using the KST methods for different sizes of DC soils

Although sequential extractions are operationally defined and not fully specific in extracting the element bound to a given fraction, they can provide comparative information to elucidate the relative contribution of the target compound and aid in the predictions of elemental mobility (Keon et al., 2001; Pueyo et al., 2003). Based on the chemical properties of the target binding phases, apportions of arsenic in mg kg_1 can be quantified with sufficient sensitivity (Keon et al., 2001). Arsenic and iron species were analyzed for each extraction step of the sequential extraction procedure that is well described by Jang et al. (2005).

Cumulative mass percentages for each size of DC soils were analyzed with the sieve analysis method, and arsenic extractions (KST methods) for different-size soils acquired from the sieve analysis were obtained. These results support the essential information for the following experimental results (especially soil size and washing agent effects on washing efficiency).

Effects of washing agents, concentrations, soil sizes, ratio of solution volume $(\mathrm{ml})$ to soil mass $(\mathrm{g})$, and sequential washing by use of single agent 
In this study, $\mathrm{HCl}$ and $\mathrm{NaOH}$ were selected as washing agents because they have been known to be effective in extracting arsenic and are economical (Van Benschoten et al., 1994; Jang et al., 2005). To estimate washing efficiencies of different concentrations of each agent on different sizes of DC soils, the following batch-scale washing tests were conducted. Fifty grams of different sizes of DC soils $(0.25-4.7,0.15-4.7,0.09-4.7$, and $<4.7 \mathrm{~mm})$ were washed with $250 \mathrm{ml}$ of predetermined concentrations $(0.1,0.2$, $0.5,1$, or $2 \mathrm{M}$ ) of $\mathrm{HCl}$ or $\mathrm{NaOH}$. The suspension was then mixed at $20 \pm 0.5 \_\mathrm{C}$ in a shaker at $300 \mathrm{rpm}$ for $6 \mathrm{~h}$. This mixing condition was identically applied for all tests. After mixing, arsenic in washing solutions was analyzed to calculate the extracted arsenic concentrations based on the mass of DC soils. Suspended solids were separated by filtration (0.45-1m micropore filter) and dried at 105 _ $\mathrm{C}$ for $2 \mathrm{~h}$ before arsenic extractions (KST methods). Through this method, we evaluated whether arsenic-contaminated soils are satisfactory for on-site disposal or reuse after washing.

Regarding the experimental results for soil size effects, nonhomogeneous soils $(2.0-4.7 \mathrm{~mm})$ and soils of target physical sizing $(<0.15 \mathrm{~mm})$ were excluded from the following experiments. The ratio of solution volume $(\mathrm{ml})$ to soil mass ( $\mathrm{g}$ ) (designated as $\mathrm{ml} \mathrm{g}_{-}{ }^{1}$ ) is a significant parameter in soil washing. Different concentrations of $\mathrm{HCl}(0.1,0.2$, or $1 \mathrm{M})$ or $\mathrm{NaOH}(0.2$ or $1 \mathrm{M})$ solution were used with different ratios $\left(1,3,5\right.$, or $\left.10 \mathrm{ml} \mathrm{g}{ }_{-}\right)$, in which soil mass was fixed at $20 \mathrm{~g}$. Five series of sequential washing tests were conducted with the same concentration of single agent each time to find the applicability of sequential washing. For these tests, different concentrations of $\mathrm{HCl}(0.1,0.2$, or $1 \mathrm{M})$ or $\mathrm{NaOH}(0.2$ or $1 \mathrm{M})$ were applied at a fixed ratio (5 ml g_1 ) of solution volume to soil mass $(20 \mathrm{~g})$.

\section{Full text is available at :}

http://www.sciencedirect.com/science/article/pii/S0045653506007181 
